\title{
HOW MANY SETS ARE POROUS?
}

\author{
TUDOR ZAMFIRESCU
}

\begin{abstract}
The notion of a $\sigma$-porous set is often used to sharpen results on sets of first Baire category or of measure zero. It essentially uses the related notion of porosity. We find out in this note that there are quite a few porous sets: In complete convex metric spaces, most totally bounded closed sets are porous! Then we strengthen this result for the case of a Banach space.
\end{abstract}

Let $(X, d)$ be a complete, convex metric space. The convexity means that for any distinct points $x, y \in X$ there exists a third point $z \in X$ such that $d(x, z)+d(z, y)=$ $d(x, y)$. It is known (see, for example, $[4$, pp. 314, 315]) that the space $(B(X), \delta)$ of all bounded closed subsets of $X$, endowed with the Hausdorff metric $\delta$, is also complete. In $B(X)$, the subset $B$ of all totally bounded closed sets $M$ (for which, by definition, $\forall \varepsilon>0, \exists$ a finite set $F \subset X$ such that $\delta(F, M)<\varepsilon$ is closed, hence $(B, \delta)$ is complete.

For $x \in X$ and $M \in B(X)$ let

$$
\begin{aligned}
& K(x, \rho)=\{y \in X: d(x, y) \leq \rho\}, \\
& K^{0}(x, \rho)=\{y \in X: d(x, y)<\rho\}, \\
& \mathcal{K}(M, \rho)=\{N \in B(X): \delta(M, N) \leq \rho\} .
\end{aligned}
$$

For $G \subset X, \bar{G}$ means the closure of $G$.

A set $M \subset X$ is called porous with coefficient $\alpha$ if $\forall x \in X$ and $\varepsilon>0, \exists y \in K(x, \varepsilon)$ such that

$$
K(y, \alpha d(x, y)) \cap M=\varnothing .
$$

A countable union of porous sets (with various coefficients) is called $\sigma$-porous. A countable union of porous sets with coefficient $\alpha$ is said to be $\alpha$-uniformly $\sigma$-porous.

We say that most (or nearly all, or $\alpha$-nearly all) elements of a Baire metric space have a certain property if those elements not enjoying it form a set of first Baire category (or a $\sigma$-porous set, or an $\alpha$-uniformly $\sigma$-porous set).

Besides the many old and new results in analysis involving most elements of various spaces, there are recent similar results in the geometry of convex bodies (for a survey see [2 or 6]). While several results on nearly all elements of certain spaces already appeared in functional analysis, [7] contains the first similar results so far in the geometry of convex bodies.

P. Gruber [3] proved in Euclidean spaces that most compact sets have measure zero. The fact that they are also nowhere dense is implicitly contained in C. Kuratowski's book [4]. So, the following natural question arises: Are most compact sets in $\mathbf{R}^{n}$ porous? The answer is yes, as the following theorems show.

Received by the editors April 12, 1986.

1980 Mathematics Subject Classification (1985 Revision). Primary 54E50, 54E52; Secondary $46 B 25$. 


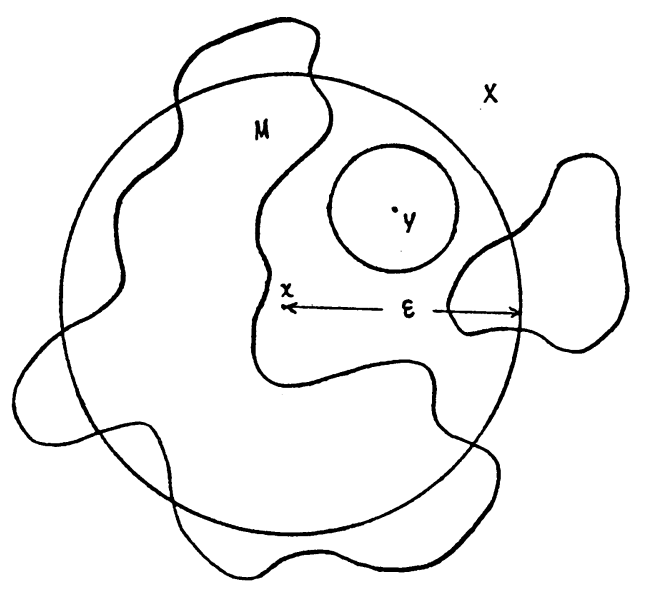

THEOREM 1. Let $(X, d)$ be a complete convex metric space. Then, for any $\alpha \in(0,1)$, most totally bounded closed sets in $X$ are porous with coefficient $\alpha$.

PROOF. Let

$$
B_{n}=\left\{M \in B: \exists y \in X \text { s.t., } \forall x \in K\left(y, n^{-1}\right), K(x, \alpha d(x, y)) \cap M \neq \varnothing\right\} .
$$

Obviously, $\bigcup_{n} B_{n}$ is the set of all elements of $B$ which are not porous with coefficient $\alpha$. Thus, to prove the theorem it suffices to show that $B_{n}$ is nowhere dense for every $n$.

Let $O$ be an open set in $B$. Consider the finite set $F \in O$. Let

$$
\xi=\min \left\{\min \{d(x, y): x, y \in F, x \neq y\}, n^{-1}\right\} \text {. }
$$

Put

$$
G=\bigcup_{x \in F} K^{0}\left(x, \frac{1-\alpha}{5-\alpha} \xi\right) .
$$

We claim that, $\forall y \in X, \exists x \in K\left(y, n^{-1}\right)$ such that

$$
K(x, \alpha d(x, y)) \cap G=\varnothing .
$$

This is obvious if $y \notin \bar{G}$. Suppose now $y \in \bar{G}$. Let $x_{0}, x_{1} \in F$ be such that

$$
d\left(y, x_{0}\right)=\min _{x \in F} d(y, x), \quad d\left(y, x_{1}\right)=\min _{x \in F \backslash\left\{x_{0}\right\}} d(y, x) .
$$

Then necessarily $y \in K\left(x_{0},(1-\alpha) \xi /(5-\alpha)\right)$.

We have

$$
d\left(y, x_{1}\right) \geq d\left(x_{0}, x_{1}\right)-d\left(y, x_{0}\right) \geq \xi-\frac{1-\alpha}{5-\alpha} \xi=\frac{4}{5-\alpha} \xi .
$$

Since $X$ is complete and convex, by Menger's theorem [5], there exists a geodesic path $P$ joining $y$ with $x_{1}$ of length $d\left(y, x_{1}\right)$. Let $x$ be the point of $P$ verifying

$$
d(x, y)=\min \left\{\frac{1}{2} d\left(y, x_{1}\right), n^{-1}\right\} \text {. }
$$

We have $d\left(x, x_{i}\right) \geq d\left(x, x_{1}\right)$ for every $x_{i} \in F$ with $i \neq 0$. Suppose, indeed, $d\left(x, x_{2}\right)<$ $d\left(x, x_{1}\right)$ for some $x_{2} \in F$. Then

$$
d\left(y, x_{2}\right) \leq d(y, x)+d\left(x, x_{2}\right)<d(y, x)+d\left(x, x_{1}\right)=d\left(y, x_{1}\right),
$$

which contradicts the choice of $x_{1}$. 
Obviously, $x \in K\left(y, n^{-1}\right)$. Suppose $z \in K(x, \alpha d(x, y)) \cap G$. Then there is an $x_{i} \in F$ such that

$$
d\left(z, x_{i}\right)<\frac{1-\alpha}{5-\alpha} \xi
$$

If $i=0$, then

$$
d(x, y) \leq d(x, z)+d\left(z, x_{0}\right)+d\left(x_{0}, y\right)<\alpha d(x, y)+\frac{1-\alpha}{5-\alpha} \xi+\frac{1-\alpha}{5-\alpha} \xi
$$

whence $d(x, y)<2 \xi /(5-\alpha)$.

Now, either $d(x, y)=\frac{1}{2} d\left(y, x_{1}\right)$, which implies $d\left(y, x_{1}\right)<4 \xi /(5-\alpha)$, thus contradicting $(*)$, or $d(x, y)=n^{-1} \geq \xi$, which yields $\xi<2 \xi /(5-\alpha)$, contradicting the choice of $\alpha$. If $i \neq 0$, then

$$
d(x, y) \leq d\left(x, x_{1}\right) \leq d\left(x, x_{i}\right) \leq d(x, z)+d\left(z, x_{i}\right)<\alpha d(x, y)+\frac{1-\alpha}{5-\alpha} \xi
$$

whence $d(x, y)<\xi /(5-\alpha)$ and either $d(x, y)=\frac{1}{2} d\left(y, x_{1}\right)$, whence $d\left(y, x_{1}\right)<$ $2 \xi /(5-\alpha)$, in contradiction with $(*)$, or else $d(x, y)=n^{-1} \geq \xi$, whence $\xi<$ $\xi /(5-\alpha)$, which again contradicts the choice of $\alpha$.

Consider now any set $M \in O$ verifying $\delta(M, F)<(1-\alpha) \xi /(5-\alpha)$. Clearly, $M \subset G$ and for every $y \in X$, there is an $x \in K\left(y, n^{-1}\right)$ such that

$$
K(x, \alpha d(x, y)) \cap M=\varnothing .
$$

Therefore $M \notin B_{n}$. Hence $B_{n}$ is nowhere dense and the theorem is proved.

THEOREM 2. In a Banach space $X$, for any $\alpha, \beta>0$ with $2 \alpha+\beta<1, \alpha$-nearly all bounded closed sets are porous with coefficient $\beta$.

ProOF. We prove that

$$
B_{n}=\left\{M \in B(X): \exists y \in X \text { such that } \forall x \in K\left(y, n^{-1}\right): K(x, \beta\|x-y\|) \cap M \neq \varnothing\right\}
$$

is porous with coefficient $\alpha$.

Let $M \in B(X)$ and $r \in \mathbf{N}$ such that $M \subset K(\mathbf{0}, 2 r)$. Then choose a support functional of $K(\mathbf{0}, 2 r)$, i.e. a nonidentically zero linear functional $f$ on $X$ such that, letting $\mu=\sup f(K(0,2 r))$, we can find a point $x_{\mu}$ in $K(0,2 r) \cap f^{-1}(\mu)$. (By the second Bishop-Phelps theorem, the support functionals of any bounded closed convex set lie densely in $X^{*}$, see [1]. In our case a simple corollary of the HahnBanach theorem suffices.) Of course,

$$
\left\|x_{\mu}\right\|=2 r, \quad f(K(\mathbf{0}, 2 r))=[-\mu, \mu] .
$$

Take $\nu_{0}=-\mu, \nu_{1}, \ldots, \nu_{n r}=0, \ldots, \nu_{2 n r}=\mu$ with $\nu_{i}-\nu_{i-1}=\mu / n r$. Let

$$
L=\bigcup_{x \in M} K\left(x, n^{-1}\right) \cap \bigcup_{i=0}^{2 n r} f^{-1}\left(\nu_{i}\right) \text {. }
$$

We claim that, for any $x \in M$, there exists $x^{*} \in K\left(x, n^{-1}\right) \cap \bigcup_{i} f^{-1}\left(\nu_{i}\right)$. Indeed, since $-\mu \leq f(x) \leq \mu$, there is an index $i$ satisfying $\nu_{i-1}<f(x) \leq \nu_{i}$. Suppose $\nu_{i}-f(x) \leq f(x)-\nu_{i-1}$; the other case is similar. We take

$$
x^{*}=x+\frac{\nu_{i}-f(x)}{\mu} x_{\mu} .
$$


Then

and

$$
\left\|x^{*}-x\right\|=\frac{\nu_{i}-f(x)}{\mu}\left\|x_{\mu}\right\| \leq \frac{\nu_{i}-\nu_{i-1}}{2 \mu} \cdot 2 r=n^{-1}
$$

Hence

$$
f\left(x^{*}\right)=f(x)+\frac{\nu_{i}-f(x)}{\mu} f\left(x_{\mu}\right)=\nu_{i} .
$$

$$
M \subset \bigcup_{x \in L} K\left(x, n^{-1}\right), \quad L \subset \bigcup_{x \in M} K\left(x, n^{-1}\right),
$$

which yield $\delta(M, L) \leq n^{-1}$.

Consider now the ball $\mathcal{K}(L, \alpha \delta(L, M))$. Since $\delta(L, M) \leq n^{-1}$, we have $\alpha \delta(L, M)$ $\leq \alpha / n$. Take $Q \in \mathcal{K}(L, \alpha \delta(L, M))$. Let

$$
S_{i}=f^{-1}\left(\left[\nu_{i}-\frac{\alpha \mu}{2 n r}, \nu_{i}+\frac{\alpha \mu}{2 n r}\right]\right)
$$

and $S=\bigcup_{i=0}^{2 n r} S_{i}$.

Then $Q \subset S$. Indeed, let $q \in Q$. There exists $x \in L$ such that $\|q-x\| \leq \alpha / n$. Let $\nu_{i}=f(x)$ and suppose $f(q)>\nu_{i}$. The "<" case is analogous and "=" is clear.

$$
f(q-x)=\frac{\alpha}{2 n r} f\left(\frac{2 n r}{\alpha}(q-x)\right),
$$

where $\|2 n r(q-x) / \alpha\| \leq 2 r$; whence $f(2 n r(q-x) / \alpha) \leq \mu$ and $f(q) \leq \nu_{i}+\alpha \mu / 2 n r$.

We now show that $Q \notin B_{n}$. Take any $y \in X$. If $y \notin S$, it suffices to remark that $Q \subset S$. If $y \in S$, then $y \in S_{i}$ for some index $i$. Assume, for instance, $f(y) \geq \nu_{i}$; the case $f(y)<\nu_{i}$ is similar. Consider

We have

$$
x=y+\frac{1}{2 n r} x_{\mu} .
$$

$$
\|x-y\|=\frac{1}{2 n r}\left\|x_{\mu}\right\|=n^{-1},
$$

hence $x \in K\left(y, n^{-1}\right)$. We prove that $K(x, \beta\|x-y\|) \cap S=\varnothing$. Let $z \in K(x, \beta\|x-y\|)$. Then $\|z-x\| \leq \beta / n$. Also,

Since

$$
f(z-x)=\frac{\beta}{2 n r} f\left(\frac{2 n r}{\beta}(z-x)\right) .
$$

we have

$$
-\mu \leq f\left(\frac{2 n r}{\beta}(z-x)\right) \leq \mu,
$$

It follows, using $2 \alpha+\beta<1$, that

$$
-\frac{\beta \mu}{2 n r} \leq f(z-x) \leq \frac{\beta \mu}{2 n r}
$$

$$
f(z) \geq f(x)-\frac{\beta \mu}{2 n r}=f(y)+\frac{1}{2 n r} f\left(x_{\mu}\right)-\frac{\beta \mu}{2 n r} \geq \nu_{i}+\frac{\mu}{2 n r}-\frac{\beta \mu}{2 n r}>\nu_{i}+\frac{\alpha \mu}{2 n r},
$$

and

$$
\begin{aligned}
f(z) & \leq f(x)+\frac{\beta \mu}{2 n r}=f(y)+\frac{1}{2 n r} f\left(x_{\mu}\right)+\frac{\beta \mu}{2 n r} \leq \nu_{i}+\frac{\mu}{2 n r}(1+\alpha+\beta) \\
& <\nu_{i}+\frac{\mu}{2 n r}(2-\alpha)=\nu_{i}+\frac{\mu}{n r}-\frac{\alpha \mu}{2 n r}=\nu_{i+1}-\frac{\alpha \mu}{2 n r}
\end{aligned}
$$

which shows that $z \notin S_{i} \cup S_{i+1}$ and therefore $z \notin S$. This completes the proof. 
THEOREM 3. In a Banach space $X$, for any $\alpha, \beta>0$ with $2 \alpha+\beta<1, \alpha$-nearly all totally bounded closed sets are porous with coefficient $\beta$.

The proof is identical to that of Theorem 2. We only have to add the obvious remark that, if $M$ is totally bounded, then $L$ is totally bounded too.

ACKNOWLEDGMENT. Many thanks are due to the referee, who kindly reported an error in the proof of Theorem 2.

\section{REFERENCES}

1. E. Bishop and R. Phelps, The support functionals of a convex set, Convexity, Proc. Sympos. Pure Math., vol. 7, Amer. Math. Soc, Providence, R.I., 1963, pp. 27-35.

2. P. Gruber, Results of Baire category type in convexity, Discrete Geometry and Convexity (New York, 1982), Ann. New York Acad. Sci., Vol. 440, New York Acad. Sci., New York, 1985, pp. 163-169.

3.

4. C. Kuratowski, Topologie. I, PWN, Warszawa, 1958.

5. K. Menger, Untersuchunger über allgemeine Metrik, Math. Ann. 100 (1928), 75-163.

6. T. Zamfirescu, Using Baire categories in geometry, Rend. Sem. Mat. Univ. Politec. Torino 43 (1985), 67-88.

7. __ Nearly all convex bodies are smooth and strictly convex, Monatsh. Math. (to appear).

FaChBereich MATHematik, Universitä́t DORTMUnd, 46 DORTMUND, Federal REPUBLIC OF GERMANY (Current address)

DEPARTMENT OF MATHEMATiCs AND COMPUTER SCIENCE, California STATE UNiversity-LOS ANGeles, 5151 State UNiversity DRIVE, LOS ANgeles, CALIFORNIA 90032 